\title{
Implementing tobacco tracking codes in an individual practice association or a network model health maintenance organisation
}

\author{
Charles J Bentz
}

The Providence Health System (PHS) in Oregon is an individual practice association model health maintenance organisation with more than 300000 covered lives in our capitated model and more than 360000 members in our preferred provider model, which is a discounted fee for service structure. PHS has 12000 employees, over 1400 acute and long term beds, and a demonstrated commitment to social accountability. Tobacco cessation programs are one of the top priorities for reducing overall cardiac morbidity and mortality. A regional health system task force for tobacco cessation comprised of key personnel and stakeholders was formed in 1994 (table 1). The physician leader's role was to educate medical care providers on smoking cessation, while the program development administrator helped coordinate activities and was instrumental in securing funds for all initiatives. Health education played a critical role in administration of the intensive cessation interventions and other member focused interventions. Health plan involvement was important in changing the benefit structure of the health plan to broaden coverage for smoking cessation programs. Pharmacy input was needed with

Smoking Cessatio and Prevention, Providence Health System, Oregon, 9205 SW Barnes Road, Suite \#25, Portland, OR 97225, USA; cbentzmd aprovidence.org C J Bentz

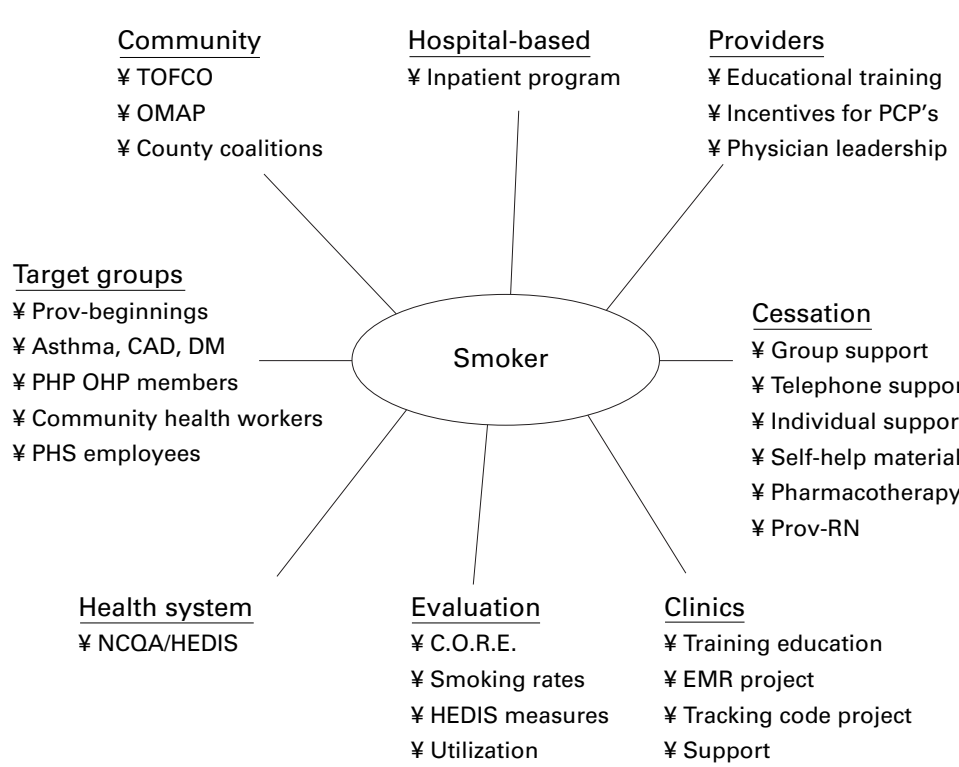

Figure 1 Providence Health System (PHS) smoking cessation and prevention program 1999. TOFCO, Tobacco Free Coalition of Oregon; OMAP, Oregon Medicaid Assistance Program; PHP, Providence Health Plan; OHP, Oregon Health Plan; NCQA, National Committee for Quality Assurance; HEDIS, Healthcare Employer Data Information Set; C.O.R.E. Centre for Outcomes Research and Education; EMR, electronic medical record; $C A D$, coronary artery disease; $D M$, diabetes mellitus; Prov- $R N$, a telephone point of service patient advice line; PCP, primary care physicians.
Table 1 Make up of PHS smoking cessation task force

\begin{tabular}{ll}
\hline - Physician leader & - Health education \\
- Program development & - Research analyst \\
- Respiratory care & - Home care \\
- Hospital administration & - Health plan \\
- Long term care & Quality management \\
- Human resources & - Care management \\
- Behavioural health & - Pharmacy \\
\hline
\end{tabular}

the advent of several new pharmaceutical tobacco cessation interventions.

The PHS smoking cessation task force developed a multifaceted approach to tobacco cessation (fig 1). The overall program starts with a variety of cessation services offered to our patients. The group support program is a 10 session behaviour modification class. The telephone support program ("Free \& Clear", Group Health Cooperative, Puget Sound) is provided to members. The individual support intervention (one on one), for highly comorbid patients who have failed group support, consists of individual counselling with a highly trained smoking cessation counsellor for 12 months. The task force developed self help materials in Spanish and Russian. We have a pharmacotherapy benefit for the over the counter nicotine patch and bupropion (Zyban; GlaxoWellcome), which is linked to participation in a structured behavioural modification program. To any interested clinic, we offer, at no cost, a tailored training program based on the 4A's developed by the National Cancer Institute, ${ }^{1}$ refined by researchers at the northwest region of Kaiser Permanente, ${ }^{2}$ which draws from the "stages of change" theory ${ }^{3}$ and "self determination" theory. ${ }^{4}$

PHS recently received full accreditation from the National Committee for Quality Assurance, and the efforts in tobacco cessation played an important role in that accreditation. Specific groups are targeted for smoking cessation, including patients with coronary artery disease, diabetes, and asthma. Members of the PHS task force have been involved in community activities, such as the Tobacco Free Coalition of Oregon, and have helped shape the state of Oregon's preventive strategy for the Oregon Medicaid Assistance Program.

We have also implemented a hospital based smoking cessation intervention based on work by Stevens et al. ${ }^{5}$ In this program, every patient admitted to a PHS hospital is asked about current smoking status by the admitting department, and the smoking status of every patient is recorded in the hospital demographic database. Lists of inpatient smokers are printed 


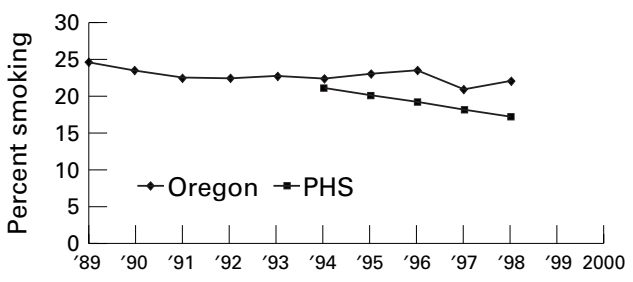

Figure 2 Smoking rates in PHS versus state of Oregon.

each day in the respiratory care departments, and dedicated respiratory therapists systematically provide a smoking cessation intervention to interested patients as a routine part of inpatient care at no additional cost to patients.

PHS funds a physician leader to champion smoking cessation within the health system. His efforts led to PHS being the first health maintenance organisation in Oregon to provide a financial incentive for primary care physicians to address tobacco cessation in routine office settings.

This multifaceted approach has lowered smoking rates among Providence Health Plan members. Smoking rates, as measured by the Oregon behavioural risk factor survey conducted by the Oregon Health Division, were compared with a PHS survey using the same methodology. Over the last four years, we have seen a significant decrease in self reported smoking rates, down to $17 \%$ in 1998 (fig 2).

There are barriers and driving forces encountered in implementing tobacco cessation in all types of managed care settings. ${ }^{6} \mathrm{We}$ confirmed that many of these barriers affect our clinics. In an evaluation of smoking cessation in one of our primary care offices we found that physicians faced time pressures, patient issues, paperwork, and lack of incentive. We also found that medical assistants were too busy, forgot about smoking, and were frustrated by lack of provider interest. A project of continuous quality improvement in one clinic simplified the medical assistant's role, gave them feedback on performance, and provided several training sessions, resulting in dramatic improvement increases in tobacco cessation.

Understanding how to build measurement into routine primary care is needed if we expect to have improvement in the delivery of preventive health care in routine office settings. A review by Nelson et al clearly laid out principles that were followed as we have developed

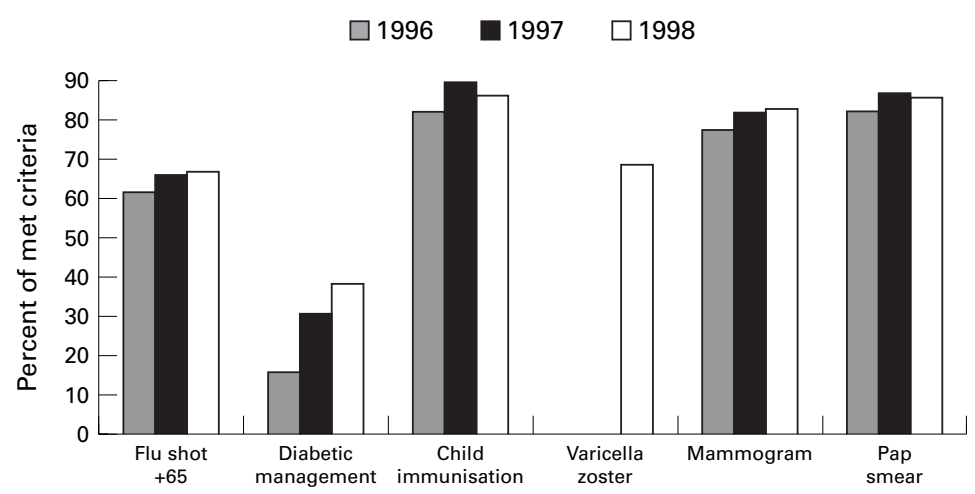

Figure 3 Quality bonus performance in PHS 1996-98.
Table 2 Building measurement into clinical practice ${ }^{7}$

- Seek usefulness, not perfection, in the measurement

- Use a balanced set of process, outcome, and cost measures

- Keep the measurement simple (think big, but start small)

- Keep the measurement simple (think

- Write down operational definitions of the measures

- Measure small, representative samples

- Build the measurement into daily work

- Develop a measurement team

a novel method of tracking and providing feedback to providers on the delivery of preventive health care (table 2).

\section{Primary care quality bonus}

We have developed a successful "primary care quality bonus", which pays primary care physicians to deliver preventive health care. A small amount of the capitated payment is withheld from the primary care providers ( $\$ 2$ per member per month). This withhold/bonus is paid to physicians according to their performance on various measures. Physicians accrue points in this bonus by achieving certain goals in access to care, patient satisfaction, and selected clinical quality measures issues. The quality bonus program has resulted in improvement in measures of preventive health care delivery (fig 3).

In order to build provider acceptance for the smoking cessation measure in the quality bonus, we have adopted a "stepped approach". This is a process by which the quality bonus measure for smoking cessation changes over a period of several years. Initially the goal of the incentive is to build preventive care infrastructure, then after we have at least $75 \%$ of our clinics reporting that the infrastructure to deliver the preventive care is in place, achieving the quality bonus will require attaining defined performance targets. In 1999, to meet criteria for the quality bonus smoking cessation measure, providers need only show that they have a systematic approach to smoking cessation in their office. This approach must be based on the 4A's: asking all patients about smoking status, and for paediatric patients, asking about exposure to second hand smoke; advising all smokers to quit; assisting interested smokers in developing a quit plan; arranging for follow up.

Physicians must document this process by submitting a written description and by demonstrating, in a sample of medical records, that the 4A's are taking place. Initially, the quality bonus will not measure the physicians' rates of performance of the 4A's. This stepped approach allows us to give an incentive for building office infrastructure before giving an incentive for specific performance targets. As we perfect our method of measurement we will gradually incorporate performance targets into the quality bonus measure for smoking cessation.

\section{Tracking codes for prevention}

Measurements of health care performance require chart review, which is costly and time consuming for both physicians and managed health care systems, and is not easily sustainable in the long run. Since most private practice physician offices lack the infrastructure to address tobacco use or other preventive 
Table 3 Tracking codes dictionary, based on HEDIS 3.0 quality measures and 1999 PHP quality bonus program

\begin{tabular}{|c|c|c|c|}
\hline \multicolumn{4}{|c|}{ Immunisations } \\
\hline TR700 & DtaP (DTP ACELL) & TR711 & DTP w/polio \\
\hline TR701 & DTP & TR712 & OPV \\
\hline TR702 & DT & TR713 & Polio (inj) \\
\hline TR703 & Tetanus & TR716 & Varicella \\
\hline TR704 & Mumps & TR718 & Tetanus and DT (adult) \\
\hline TR705 & Measles & TR719 & Diphtheria \\
\hline TR706 & Rubella & TR720 & DTP/HIB \\
\hline TR707 & MMR & TR724 & Influenza \\
\hline TR708 & Measles and rubella & TR732 & Pneumococcal \\
\hline TR709 & Rubella and mumps & TR737 & HIB \\
\hline TR710 & MMRV & TR724 & Influenza \\
\hline TR732 & Pneumococcal & TR721 & Prior $\mathrm{Hx}$ varicella \\
\hline \multicolumn{2}{|c|}{ Diabetic indicators } & \multicolumn{2}{|c|}{ Haemoglobin A1c level (g/dl) } \\
\hline TR800 & Dilated retinal exam & TR203 & $\geqslant 9.0$ \\
\hline TR801 & Foot exam & TR202 & $\geqslant 8<9.0$ \\
\hline TR220 & Negative both micro \& macroalbuminuria & TR201 & $\geqslant 7<8.0$ \\
\hline TR221 & Positive either micro \& macroalbuminuria & TR200 & $<7.0$ \\
\hline \multicolumn{2}{|c|}{ Smoking indicators } & \multicolumn{2}{|c|}{ Counselling } \\
\hline TR001 & Smoking & TR100 & Smoking cessation \\
\hline TR002 & Non-smoker/remote quitter ( $>6$ months) & TR101 & Substance abuse \\
\hline TR003 & Recent quitter ( $<6$ months) & TR102 & Sexually transmitted disease \\
\hline \multicolumn{2}{|c|}{ Disease management/prevention } & \multicolumn{2}{|c|}{ LDL cholesterol $(\mathrm{mg} / \mathrm{dl})$} \\
\hline TR600 & Cholesterol and lipid drug management & TR232 & $>160$ \\
\hline TR601 & Beta blocker after MI & TR231 & $>130 \leqslant 160$ \\
\hline TR602 & Aspirin use & TR230 & $\geqslant 100 \leqslant 130$ \\
\hline TR603 & ACE inhibitor use & TR229 & $<100$ \\
\hline TR301 & Lead screening questionnaire & & \\
\hline \multicolumn{4}{|c|}{ Women's health } \\
\hline TR520 & Mammography & & \\
\hline TR521 & Pap (cervical) smear & & \\
\hline TR010 & Prior total hysterectomy & & \\
\hline TR011 & Bilateral mastectomy & & \\
\hline TR500 & Prenatal visit & & \\
\hline TR510 & Postnatal visit & & \\
\hline
\end{tabular}

Measures in bold are part of the PHS system 1999 tracking codes pilot initiative.

health care systematically, a new approach is needed. We have developed a set of tracking codes, similar to the current procedural terminology codes, which allow health care providers and managed care organisations (MCOs) to measure and reimburse for delivery of these services. ${ }^{8}$ These prevention codes are documented at the point of medical care by the physician, captured on fee slips, and submitted to the MCO's claims system. It is equally important to track the exceptions to preventive health care. For example, diabetics who are on angiotensin converting enzyme (ACE) inhibitors can be excluded from needing annual screening for diabetic proteinuria. A preliminary "dictionary" of prevention tracking codes has been developed (table 3), which includes a set of codes to track tobacco use and provider advice to quit. A project to refine and implement these tracking codes has been funded by a planning grant from the Robert Wood Johnson Foundation (RWJF). The results of this project, being piloted in offices using both paper based and electronic medical records, will be available at the RWJF's "Addressing Tobacco in Managed Care" conference in February 2000.

Important concepts have emerged from the work of the PHS task force on smoking cessation. Teamwork has contributed to the success of this task force, which has received two special PHS teamwork awards (in 1997 and again in 1999). No other group in our health system has won this award twice. Defining the roles of doctors and administrators has been crucial to our success. If physicians are expected to deliver the 4A's of smoking (ask, advise, assist, arrange), then it is necessary to enable providers of health care to carry this out.

- If providers are expected to ask every patient about tobacco use, the MCO should make this possible by having a clear policy in place and supporting the development of the infrastructure to carry this out and achieve this goal.

- If providers are expected to give cessation advice to all smokers, then the MCO needs to create an incentive that encourages cessation advice and makes providers accountable.

- If physicians are expected to assist interested smokers in quitting, then the MCO needs to provide training in smoking cessation and also provide the resources needed to help highly addicted patients to quit.

- If medical care providers are expected to arrange for follow up visits for smoking cessation, then the MCO needs to help staff improve performance of this important aspect of cessation and allow appropriate compensation to occur.

\section{Conclusion}

Important concepts have evolved from our efforts to address tobacco use in our loosely affiliated health care system. Everyone benefits from improvement in the delivery of preventive health care. To make progress in this area, and smoking cessation in particular, we need to be clear about who the real customer is. While the patient is the ultimate customer, the immediate customers are physicians and the physicians' office staff. Interventions that attempt to 
improve quality of care must support infrastructure. We have demonstrated that our primary care quality bonus has improved delivery of preventive health care service, and we hope it will eventually improve the health of our patients. Doctors are benefiting from enhanced revenue collections in the form of quality bonus incentives. Our health system will save expenditures by avoiding chart review, will benefit from improved public image, and will continue to garner praise from external reviewing organisations, such as the National Committee for Quality Assurance. Simply managing care or capitating payments to physicians does not automatically improve quality of care. It takes teamwork. But if there is one thing that managed care can do, and has the promise to do, it is to improve delivery of preventive health care services.

1 US Department of Health and Human Services. How to help your patients stop smoking. A National Cancer Institute manual for physicians. Bethesda, Maryland: National Can1991 (NIH Publication No 92-3064).

2 Lichtenstein E, Hollis JF, Severson HH, et al. Tobacco cessation interventions in health care settings: rationale, model, outcomes. Addictive Behav 1996;21:709-20.

3 Prochaska JO, DiClemente CC, Norcross JC. In search of how people change: applications to addictive behaviors. American Psychologist 1992;47:1102-14.

4 Williams GC, Quill TE, Deci E, et al. The facts concerning the recent carnival of smoking in Connecticut and elsewhere. Ann Intern Med 1991;115:59-63.

5 Stevens VJ, Flasgow RE, Hollis JF, et al. A smoking cessation intervention for hospital patients. Med Care 1993;31:6572.

6 Davis RM. An overview of tobacco measures. Tobacco Control 1998; 7(suppl):S36-40, S47-9.
7 Nelson EC, Splaine ME, Batalden PB, et al. Building measurement and data collection into medical practice. Ann Intern Med 1998;128:460-6.

8 Powers M. "Tracking codes" may increase compliance with HEDIS. Capitation Management Report 1999;6:17-20.

\section{Questions and answers}

Q: Why do you use incentives for groups instead of individual physicians?

A: It is a team effort to provide preventive programs. Thus, you really need to have team rewards.

Q: Most of the large health organisations are losing substantial amounts of money, thus incentives may be one of the first things to eliminate. Do you think that is the case?

A: In our MCO, there are several funds. There is the institutional fund, which pays the hospital, the referral fund which pays the providers, and the PCP fund which goes to pay primary care. Every single major fund is in a deficit right now. So, there is a huge outcry and it is a time of turmoil. If there are funding crises, what better time and way to figure out what is important? You can become an advocate and go to the chief executive officer and say, "This is a great opportunity for us to show what is most important about managed care-which is prevention, all types of prevention. These bonuses are important because it is how we are being measured; they demonstrate the quality of our organization". These are very effective arguments and can help. 